\title{
Divergence et parallélisme des rayons d'étirement cylindriques
}

\author{
Guillaume ThÉRET
}

\begin{abstract}
Une ligne d'étirement cylindrique est une ligne d'étirement au sens de Thurston dont la lamination horocyclique est une multicourbe pondérée. Nous montrons ici que deux lignes cylindriques correctement paramétrées sont parallèles si et seulement si ces lignes convergent vers le même point du bord de Thurston de l'espace de Teichmüller.

A cylindrical stretch line is a stretch line, in the sense of Thurston, whose horocyclic lamination is a weighted multicurve. In this paper, we show that two correctly parameterized cylindrical lines are parallel if and only if these lines converge towards the same point in Thurston's boundary of Teichmüller space.
\end{abstract}

30F60, 57M50, 53C22

\section{Introduction}

Fixons une surface fermée orientable $\Sigma$ de genre fini supérieur ou égal à deux. Notons $\mathcal{T}(\Sigma)$ l'espace de Teichmüller associé à la surface $\Sigma$. Dans cet article, nous munissons $\mathcal{T}(\Sigma)$ de la métrique asymétrique de Thurston $d_{\mathcal{T}}$. Cette métrique est donnée par la formule

$$
d_{\mathcal{T}}(g, h)=\log \sup _{\alpha \in \mathcal{M} \mathcal{L}(\Sigma)} \frac{\ell_{h}(\alpha)}{\ell_{g}(\alpha)}
$$

où $\mathcal{M L}(\Sigma)$ désigne l'espace des laminations géodésiques mesurées et $\ell_{h}(\alpha)$ désigne la longueur de la lamination géodésique mesurée $\alpha$ pour la classe d'isotopie $h \in \mathcal{T}(\Sigma)$ de métriques hyperboliques. L'adjectif "asymétrique" insiste sur le fait qu'il existe des points $g, h$ de $\mathcal{T}(\Sigma)$ pour lesquels $d_{\mathcal{T}}(g, h) \neq d_{\mathcal{T}}(h, g)$.

Une ligne d'étirement de Thurston est une géodésique orientée $t \mapsto h_{t}$ de l'espace de Teichmüller pour la métrique de Thurston, c'est-à-dire que l'on a, pour tout $s \leq t$,

$$
d_{\mathcal{T}}\left(h_{s}, h_{t}\right)=t-s .
$$

Dans cet article, toutes les paramétrisations seront positives (ie, préserveront l'orientation) et par la longueur d'arc. 
Une ligne d'étirement est déterminée par deux laminations géodésiques, l'une étant complète, appelée le support de la ligne d'étirement et généralement notée $\mu$, et l'autre étant la classe projective d'une lamination mesurée totalement transverse à $\mu$, appelée la direction de la ligne d'étirement. La souche d'une ligne d'étirement est la souslamination du support de la ligne qui possède une mesure transverse de support total et qui est maximale au sens de l'inclusion.

Une multicourbe est une réunion de courbes simples fermées disjointes. La donnée d'une mesure transverse sur une multicourbe est équivalente à la donnée d'une pondération positive des composantes de cette multicourbe.

Dans cet article, nous imposerons aux supports des lignes d'étirement d'être des laminations récurrentes par chaînes, c'est-à-dire limites de multicourbes pour la topologie de Hausdorff.

On dira d'une ligne d'étirement qu'elle est cylindrique si sa direction est la classe projective d'une multicourbe pondérée.

Nous dirons de deux lignes d'étirement $t \mapsto g_{t}, t \mapsto h_{t}$, qu'elles divergent si les distances $d_{\mathcal{T}}\left(g_{t}, h_{t}\right)$ et $d_{\mathcal{T}}\left(h_{t}, g_{t}\right)$ tendent vers l'infini lorsque $t$ tend vers $+\infty$. Enfin, nous dirons de ces lignes qu'elles sont parallèles si, quitte à changer la paramétrisation, les distances précédentes restent bornées pour $t$ assez grand (nous verrons un peu plus loin que la possibilité de changement de paramétrisation dans cette définition est importante, contrairement au cas des métriques symétriques).

Le but de ce papier est de d'établir le résultat suivant.

Théorème Deux lignes d'étirement cylindriques, de supports récurrents par chaînes, sont parallèles si et seulement si elles ont même direction.

Nous démontrerons en fait quelque chose d'un peu plus précis, à savoir, que deux lignes d'étirement cylindriques non parallèles divergent, quitte à les reparamétrer.

Notre résultat est en contraste avec la situation où l'espace de Teichmüller est muni de la métrique de Teichmüller. Dans ce cas, H Masur [2] a montré l'existence de lignes d'étirement de Teichmüller cylindriques (dites de Jenkins-Strebel dans ce contexte) dirigées par des classes projectives distinctes mais néanmoins parallèles.

Le fait qu'il faille éventuellement changer la paramétrisation des lignes d'étirement vient du résultat suivant, qui sera démontré dans cet article.

Théorème Soit $t \mapsto h_{t}$ une ligne d'étirement cylindrique de support récurrent par chaînes. Pour tout nombre strictement positif $c$, on a

$$
\forall t, d_{\mathcal{T}}\left(h_{t}, h_{t+c}\right)=c, \quad \text { et } \quad \lim _{t \rightarrow \infty} d_{\mathcal{T}}\left(h_{t+c}, h_{t}\right)=\infty
$$


En effet, ce résultat montre que si l'on ne prend pas garde à reparamétrer la ligne, deux lignes d'étirement dont les images coïncident pourraient ne pas être parallèles. Notez que deux lignes d'étirement divergentes ne sont pas parallèles.

Remerciements Je tiens à remercier chaleureusement mon relecteur, pour son travail et pour ses remarques pertinentes qui ont permis d'améliorer cet article.

\section{Préliminaires}

Nous rappelons ici très succinctement la construction des lignes d'étirement de Thurston [6].

Fixons une lamination géodésique complète $\mu$ ainsi qu'une métrique hyperbolique $h$ sur $\Sigma$. Chaque composante de $\Sigma \backslash \mu$ est l'intérieur d'un triangle idéal. On construit d'abord un feuilletage partiel de l'intérieur de chaque triangle idéal de $\Sigma \backslash \mu$. Les feuilles de ce feuilletage partiel sont des arcs d'horocycles centrés aux sommets du triangle. Le feuilletage est invariant par la symétrie d'ordre 3 de chaque triangle. Une région triangulaire bordée par 3 arcs d'horocycles de longueur 1 reste non-feuilletée. On étend ensuite par continuité ce feuilletage partiel défini sur $\Sigma \backslash \mu$ en un feuilletage partiel sur $\Sigma$. Il y a alors exactement une région non-feuilletée par triangle idéal de $\mu$. On munit ce feuilletage partiel d'une mesure transverse en décrétant que la mesure d'un arc compact transverse est la longueur de sa projection le long des feuilles du feuilletage partiel sur une feuille de $\mu$. On note ce feuilletage partiel mesuré $F_{\mu}(h)$ et on l'appelle le feuilletage horocyclique associé à $\mu$ et $h$. La classe, au sens des feuilletages mesurés, du feuilletage horocyclique est notée de la même façon.

Thurston a montré que la construction précédente,

$$
F_{\mu}: \mathcal{T}(\Sigma) \rightarrow \mathcal{M F}(\Sigma), \quad h \mapsto F_{\mu}(h),
$$

est un homéomorphisme sur son image. Cette image est constituée, dans le cas des surfaces fermées, des classes de feuilletages mesurés transverses à $\mu$.

La ligne d'étirement de support $\mu$ et passant par $h \in \mathcal{T}(\Sigma)$ est la courbe

$$
t \mapsto h_{t}=F_{\mu}^{-1}\left(e^{t} F_{\mu}(h)\right),
$$

où $t$ varie dans $\mathbb{R}$ et où la notation $e^{t} F_{\mu}(h)$ signifie que l'on a multiplié la mesure transverse du feuilletage horocyclique $F_{\mu}(h)$ par $e^{t}$. Notez que, par définition,

$$
h_{0}=h \quad \text { et } \quad F_{\mu}\left(h_{t}\right)=e^{t} F_{\mu}(h) .
$$


L'image, par l'application $F_{\mu}$, de la ligne d'étirement passant par $h$ et de support $\mu$ est l'ensemble $\left\{e^{t} F_{\mu}(h): t \in \mathbb{R}\right\}$. Tous les points d'une ligne détirement définissent des feuilletages horocycliques qui appartiennent à la même classe projective, qu'on appelle la direction de la ligne d'étirement. Ainsi, une ligne d'étirement, comme sous-ensemble de $\mathcal{T}(\Sigma)$, est déterminée par son support et sa direction.

Dans la suite, nous parlerons également de la lamination horocyclique $\lambda_{\mu}(h)$ qui n'est rien d'autre que la lamination géodésique mesurée associée au feuilletage horocyclique $F_{\mu}(h)$.

\section{3 Étirements élémentaires cylindriques et longueur de la la- mination horocyclique}

Le but de cette partie est d'établir les résultats présentés plus haut dans le cas particulier où les supports des lignes d'étirements sont des laminations géodésiques élémentaires. Une lamination géodésique est élémentaire si elle est complète, récurrente par chaînes et si sa souche est une multicourbe. De manière équivalente, une lamination élémentaire est une lamination géodésique complète composée d'un nombre fini de feuilles fermées et d'un nombre fini de feuilles infinies, isolées, spiralant autour de chaque feuille fermée de telle manière que, pour un observateur situé sur la feuille fermée $a$ et regardant les spirales, celles-ci tournent dans la même direction pour les deux côtés de $a$.

Soit $\mu$ une lamination géodésique complète élémentaire de souche $\gamma$. Supposons que la structure hyperbolique $h \in \mathcal{T}(\Sigma)$ soit choisie de telle sorte que la lamination horocyclique $\lambda_{\mu}(h)$ - que nous noterons desormais $\lambda$ pour faire court - soit une multicourbe, ou, pour reprendre le langage de l'introduction, de telle sorte que la ligne d'étirement de support $\mu$ et de direction la classe de $\lambda$ soit cylindrique.

Chaque composante $\lambda_{j}, j \in\{1, \ldots, M\}$, de $\lambda$ définit un cylindre $C_{j}$ qui est la clôture de la réunion des feuilles du feuilletage horocyclique $F_{\mu}(h)$ qui sont isotopes à $\lambda_{j}$. La géodésique fermée simple $\lambda_{j}$ est appelée le cœur du cylindre $C_{j}$. Chacun des deux bords du cylindre est une feuille fermée singulière, ce qui signifie qu'elle contient un nombre fini de côtés de régions non-feuilletées du feuilletage horocyclique. Nous dirons que ces régions non-feuilletées sont adjacentes au cylindre. Par définition de la mesure transverse de $F_{\mu}(h)$, les feuilles de $\mu \cap C_{j}$ ont toutes même longueur, $w_{j}$. Le nombre $w_{j}$, qu'on appellera la largeur du cylindre $C_{j}$, est aussi le poids de la composante $\lambda_{j}$ vue comme lamination géodésique mesurée.

Nous allons donner un équivalent asymptotique de la longueur de la géodésique $\lambda_{j}$ lorsqu'on étire le long de $\mu$ indéfiniment. 
Proposition 3.1 Soit $t \mapsto h_{t}$ une ligne d'étirement élémentaire cylindrique de support $\mu$, passant par $h=h_{0}$. La lamination horocyclique $\lambda_{\mu}(h)$ est la multicourbe $\lambda=$ $\lambda_{1} \cup \cdots \cup \lambda_{M}$ avec les pondérations $w_{1}, \ldots, w_{M}$. Lorsque $t$ tend vers $+\infty$, la longueur $\ell_{h_{t}}\left(\lambda_{j}\right)$ de la courbe simple fermée $\lambda_{j}$ est équivalente à la quantité

$$
2 \sqrt{K_{j}} e^{-e^{t} w_{j} / 2},
$$

où $K_{j} \in \mathbb{N}$ est le nombre de régions non-feuilletées adjacentes à un bord du cylindre de cœur $\lambda_{j}$ multiplié par le nombre de régions non-feuilletées adjacentes à l'autre bord.

\subsection{Calculs dans un cylindre}

Soit $C$ un cylindre parmi les cylindres $C_{1}, \ldots, C_{M}$. Notons $w$ sa largeur. La multicourbe $\gamma$ traverse le cylindre $C$ en un nombre fini de segments. Au voisinage de chacun de ces segments, les feuilles isolées de $\mu$ spiralent dans un sens qui est le même de chaque côté du segment.

Une bande de $C$ désignera une partie obtenue en coupant le cylindre $C$ le long de deux feuilles de $\mu \cap C$, de telle sorte que toutes les feuilles isolées de $\mu$ à l'intérieur de cette bande ont le même sommet idéal.

Ainsi, une bande est isométrique à un rectangle du demi-plan hyperbolique, dont les bords horizontaux sont des horocycles centrés en l'infini et les bords verticaux des géodésiques asymptotes à l'infini. Dans cette description, les feuilles de $\mu$ traversant $C$ correspondent à des géodésiques verticales asymptotes à l'infini. La restriction du feuilletage horocyclique correspond quant à lui au feuilletage du rectangle par des segments euclidiens horizontaux, c'est-à-dire par des arcs d'horocycles centrés à l'infini. Les longueurs de ces arcs d'horocycles sont strictement décroissantes à mesure que l'on se rapproche linéairement du sommet idéal. L'épaisseur d'une bande est la longueur du plus grand arc d'horocycle.

Une bande est dite minimale s'il n'existe pas de bande strictement contenue en elle. Une bande minimale est donc la partie d'un triangle idéal de $\mu$ comprise entre deux feuilles du feuilletage horocyclique.

Une bande est dite maximale s'il n'existe pas de bande la contenant strictement. Il existe une unique décomposition du cylindre $C$ en bandes maximales d'intérieurs disjoints. On l'appelle la décomposition de $C$ en bandes. Les arcs d'horocycles de deux bandes adjacentes ont des convexités de signes opposés (voir la figure 1). Notez que la décomposition en bandes contient un nombre pair de bandes.

Notons $B_{1}, \ldots, B_{2 N}$ la décomposition de $C$ en bandes, avec $B_{j}$ adjacente à $B_{j+1}$. Notons $a_{1}, \ldots, a_{2 N}$ les épaisseurs respectives de ces bandes. Orientons les feuilles 


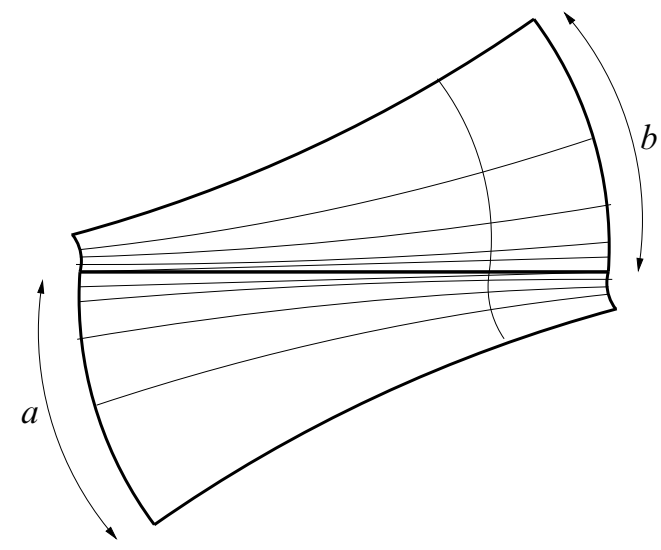

FIGURE 1: Ce dessin représente deux bandes adjacentes de la décomposition en bandes d'un cylindre. La frontière commune dans ce dessin est une composante de $\gamma \cap C$ et est représentée horizontalement, en trait épais. Une feuille intérieure du feuilletage horocyclique a été dessinée, en trait fin; elle est obtenue en concaténant deux arcs d'horocycles de convexités de signes opposés. Les épaisseurs des bandes sont notées $a$ et $b$.

de $\mu \cap C$ de telle sorte que les arcs d'horocycles représentant les épaisseurs d'indices impairs se trouvent dans le bord gauche de $C$ et les arcs d'horocycles représentant les épaisseurs d'indices pairs se trouvent dans le bord droit de $C$. Posons

$$
a_{\text {pair }}=\sum_{k=1}^{N} a_{2 k} \quad \text { et } \quad a_{\text {impair }}=\sum_{k=1}^{N} a_{2 k-1} .
$$

On paramètre l'ensemble des feuilles du feuilletage horocyclique de $C$ par la distance, par rapport au bord droit de $C$, à laquelle elles intersectent n'importe quelle feuille de $\mu \cap C$. On note $h^{*}(d)$ la longueur de la feuille fermée du feuilletage horocyclique située à la distance $d \in[0, w]$. La longueur $h^{*}(d)$ est la somme des longueurs des arcs d'horocycles contenus dans les bandes. L'ordre de ces horocycles est sans incidence sur cette longueur et l'on peut donc supposer que $h^{*}(d)$ est la longueur d'une concatenation de deux arcs d'horocycles de convexités inversées contenus dans deux bandes d'épaisseurs $a_{\text {pair }}=\sum_{k=1}^{N} a_{2 k}$ et $a_{\text {impair }}=\sum_{k=1}^{N} a_{2 k-1}$. On a

$$
h^{*}(d)=a_{\text {pair }} e^{-d}+a_{\text {impair }} e^{-w+d} .
$$

On recherche le minimum $h^{*}$ de cette function lorsque $d$ varie dans $[0, w]$. En regardant où la dérivée s'annule, on obtient que le minimum est unique, atteint pour $e^{d}=$ 
$\sqrt{a_{\text {pair }} / a_{\text {impair }}} e^{w / 2}$ et vaut

$$
h^{*}=2 \sqrt{a_{\text {pair }} a_{\text {impair }}} e^{-w / 2} .
$$

Notons $h$ la longueur de l'arc géodésique joignant le segment de $\mu \cap C$ séparant $B_{1}$ et $B_{2 N}$ à lui-même perpendiculairement. On appelle cette longueur une hauteur du cylindre $C$.

On se réfère à la figure 2. Coupons le cylindre $C$ le long de la feuille séparant $B_{1}$ et $B_{2 N}$. On place le cylindre coupé dans le demi-plan supérieur hyperbolique comme indiqué, de telle sorte qu'un des côtés géodésiques soit contenu dans la verticale issue de $O$ à partir du point d'ordonnée 1. Le nombre $h$ est la longueur de l'arc de cercle joignant perpendiculairement la verticale issue de $O$ et le demi-cercle passant par les points situés sur l'axe des abscisses aux valeurs $x_{2 N-1}$ et $x_{2 N}$. Le bord gauche de $C$, situé en bas, est la concaténation des arcs d'horocycles de longueurs $a_{1}, a_{2} e^{-w}, a_{3}$, $a_{4} e^{-w}, \ldots, a_{2 N-1}, a_{2 N} e^{-w}$. Le bord droit de $C$, situé en haut, est la concaténation des arcs d'horocycles de longueurs $a_{1} e^{-w}, a_{2}, a_{3} e^{-w}, a_{4} \cdots, a_{2 N-1} e^{-w}, a_{2 N}$. La bande $B_{j}, j \geq 1$, est bordée par les géodésiques joignant l'abscisses $x_{j-1}$ aux abscisses $x_{j-2}$ et $x_{j}\left(\operatorname{avec} x_{0}=\infty\right.$ et $\left.x_{-1}=0\right)$. Remarquons tout d'abord que l'on a

$$
x_{2 j}=a_{1}+\frac{1}{a_{2} e^{-w}+\frac{1}{a_{3}+\frac{1}{\ddots \frac{1}{a_{2 j-1}+\frac{1}{a_{2 j} e^{-w}}}}}},
$$

ce que l'on note

$$
x_{2 j}=\left[a_{1}, a_{2} e^{-w}, \ldots, a_{2 j} e^{-w}\right] \text { et } x_{2 j-1}=\left[a_{1}, a_{2} e^{-w}, \ldots, a_{2 j-1}\right] .
$$

En effet, l'isométrie qui envoie la géodésique verticale issue de 0 sur la géodésique joignant les abscisses $x_{j-1}$ et $x_{j}$ est donnée par la composition $P_{1} \circ P_{2} \circ \cdots \circ P_{j}(0)$ 
des isométries de type parabolique

$$
P_{k}: z \mapsto \begin{cases}z+a_{k} & \text { si } k \text { est impair, } \\ 1 /\left(\bar{a}_{k}+1 / z\right) & \text { si } k \text { est pair, où } \bar{a}_{k}=a_{k} e^{-w} .\end{cases}
$$

Notez que l'ordre de composition est inversé. Le point $x_{j}$ est l'image de zéro ou de l'infini par ce produit d'isométries, suivant la parité de $j$. Plus précisément, pour $j \in\{1, \ldots, N\}$,

$$
x_{2 j-1}=P_{1} \circ P_{2} \circ \cdots \circ P_{2 j-1}(0) \text { et } x_{2 j}=P_{1} \circ P_{2} \circ \cdots \circ P_{2 j}(\infty) .
$$

Soit $\theta$ l'angle à l'origine entre la verticale issue de l'origine et la courbe équidistante tangente à la géodésique joignant les abscisses $x_{2 N-1}$ et $x_{2 N}$. Un peu de géométrie hyperbolique permet d'établir

$$
\cosh (h)=\frac{1}{\cos (\theta)}
$$

En effet, si l'on paramétrise l'arc de cercle $x^{2}+y^{2}=1$ à l'aide de l'abscisse $x$, on obtient

$$
h=\int_{0}^{\sin \theta} \frac{\sqrt{d y^{2}(x)+d x^{2}}}{y(x)}=\int_{0}^{\sin \theta} \frac{\sqrt{x^{2} / y(x)^{2}+1}}{y(x)} d x=\int_{0}^{\sin \theta} \frac{d x}{1-x^{2}} .
$$

Donc $\tanh (h)=\sin (\theta)$. On en déduit la formule.

Revenons maintenant à la figure de départ. On a

$$
\cos (\theta)=\frac{x_{2 N}-x_{2 N-1}}{x_{2 N}+x_{2 N-1}}
$$

Finalement, on obtient

$$
\cosh (h)=1+\frac{2}{x_{2 N} / x_{2 N-1}-1}
$$

\subsection{Estimation asymptotique le long d'une ligne étirement}

Déformons maintenant la structure hyperbolique $h$ en l'étirant le long de $\mu$. Nous allons donner une estimation asymptotique de la longueur des composantes $\lambda_{j}$. On reprend les notations du paragraphe précédent mais on ajoute le paramètre $t$. Notons $\ell(t)$ la longueur du cœur géodésique du cylindre $C$. On a l'encadrement

$$
h(t) \leq \ell(t) \leq h^{*}(t) .
$$

La longueur de tout arc d'horocycle contenu dans la pointe d'un triangle idéal est inférieure ou égale à 1 . Elle vaut 1 si et seulement si l'arc d'horocycle est un côté d'une 


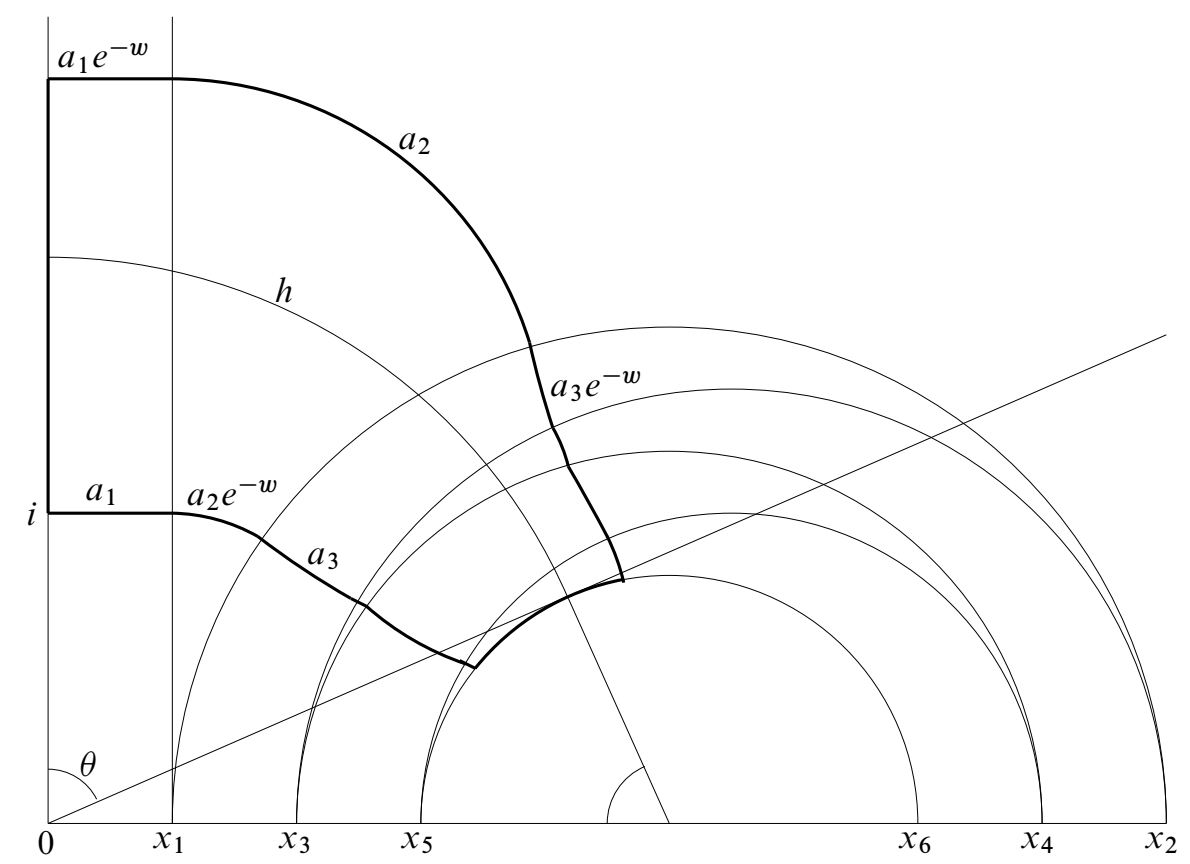

FIgURE 2: Ce dessin représente le cylindre $C$ (coupé le long d'une feuille de $\mu$ ) et sa décomposition en bandes $B_{1}, \ldots, B_{2 N}$, dans le demi-plan supérieur hyperbolique. Nous avons indiqué les épaisseurs des bandes ainsi que les abscisses $x_{1}, x_{2}, \ldots$

région non-feuilletée du feuilletage horocyclique. Lorsqu'on effectue un étirement de temps $t \geq 0$, cette longueur est élevée à la puissance $e^{t}$. Ainsi, la longueur d'un tel arc d'horocycle converge vers zéro lorsque $t$ tend vers l'infini si et seulement si elle est strictement plus petite que 1 . Sinon, elle reste constante égale à 1 . On en conclut que l'épaisseur d'une bande est une fonction de $t$ décroissante et qu'elle a pour limite, lorsque $t$ tend vers l'infini, le nombre de régions non-feuilletées adjacentes à la bande.

Nous allons maintenant donner un équivalent de $h(t)$ lorsque $t$ tend vers l'infini.

Rappelons d'abord que deux grandeurs $A$ et $B$, dépendantes de la variable réelle $t$, sont équivalentes lorsque $t$ tend vers l'infini, et nous notons cette propriété $A \sim B$, lorsque le rapport $A / B$ est bien défini pour tout $t$ positif assez grand et converge vers 1 lorsque $t$ tend vers $+\infty$.

Notons ensuite que, par définition de la mesure transverse du feuilletage horocyclique, on a $w(t)=e^{t} w$ et qu'en particulier $w(t)$ tend vers $+\infty$ lorsque $t$ tend vers l'infini. 
Lemme 3.2 On a, lorsque $t$ tend vers l'infini, les équivalences suivantes

$$
x_{2 N-1}(t) \sim a_{\text {impair }} \quad \text { et } \quad x_{2 N}(t) \sim \frac{1}{a_{\text {pair }}} e^{e^{t} w} .
$$

Démonstration Commençons cette démonstration par établir la propriété suivante:

Soient $a(t)$ et $b(t)$ deux fonctions réelles strictement positives de la variable réelle $t$. Si $b(t) \sim 1 / c(t)$ alors $[a(t), b(t)] \sim a(t)+c(t)$.

La preuve est une simple vérification: on forme le rapport $[a(t), b(t)] /(a(t)+c(t))$ et on écrit

$$
\begin{aligned}
\frac{[a(t), b(t)]}{a(t)+c(t)} & =\frac{a(t)+1 / b(t)}{a(t)+c(t)}=1+\frac{1 / b(t)-c(t)}{a(t)+c(t)} \\
& =1+\frac{c(t)(1 /(c(t) b(t))-1)}{a(t)+c(t)} \\
& =1+\frac{c(t)}{a(t)+c(t)}\left(\frac{1}{c(t) b(t)}-1\right) \\
& =1+\frac{1}{1+a(t) / c(t)}\left(\frac{1}{c(t) b(t)}-1\right) .
\end{aligned}
$$

Par hypothèse,

et

$$
\lim _{t \rightarrow+\infty} \frac{1}{c(t) b(t)}-1=0
$$

$$
0<\frac{1}{1+a(t) / c(t)} \leq 1
$$

On conclut que le rapport $[a(t), b(t)] /(a(t)+c(t))$ converge vers 1 , ce qu'il fallait démontrer.

Rappelons maintenant que pour tout $k \in\{1,2,3, \cdots\}$, les fonctions $a_{k}(t)$ sont strictement positives et majorées. Par conséquent, on a

et

$$
\begin{aligned}
{\left[a_{2 j-1}(t), a_{2 j}(t) e^{-w(t)}\right] } & \sim \frac{1}{a_{2 j}(t)} e^{w(t)} \\
{\left[a_{2 j-2}(t) e^{-w(t)}, a_{2 j-1}(t)\right] } & \sim \frac{1}{a_{2 j-1}(t)} .
\end{aligned}
$$

Pour trouver un équivalent de $\left[a_{2 j-2}(t) e^{-w(t)}, a_{2 j-1}(t), a_{2 j}(t) e^{-w(t)}\right]$, on remarque d'abord que

$$
\begin{aligned}
{\left[a_{2 j-2}(t) e^{-w(t)}, a_{2 j-1}(t), a_{2 j}(t) e^{-w(t)}\right] } & \\
& =\left[a_{2 j-2}(t) e^{-w(t)},\left[a_{2 j-1}(t), a_{2 j}(t) e^{-w(t)}\right]\right] .
\end{aligned}
$$

Algebraic 83 Geometric Topology, Volume 10 (2010) 
On utilise ensuite la propriété démontrée plus haut avec l'équivalent trouvé pour $\left[a_{2 j-1}(t), a_{2 j}(t) e^{-w(t)}\right]$, ce qui donne

$$
\left[a_{2 j-2}(t) e^{-w(t)}, a_{2 j-1}(t), a_{2 j}(t) e^{-w(t)}\right] \sim\left(a_{2 j-2}(t)+a_{2 j}(t)\right) e^{-w(t)} .
$$

De la même manière, on obtient

$$
\left[a_{2 j-3}(t), a_{2 j-2}(t) e^{-w(t)} a_{2 j-1}(t)\right] \sim a_{2 j-3}(t)+a_{2 j-1}(t) .
$$

En appliquant le même procédé, on en déduit

$$
\begin{aligned}
{\left[a_{2 j-3}(t), a_{2 j-2}(t) e^{-w(t)}, a_{2 j-1}(t), a_{2 j}(t) e^{-w(t)}\right] } & \\
& \sim a_{2 j-3}(t)+\left(a_{2 j-2}(t)+a_{2 j}(t)\right)^{-1} e^{w(t)} .
\end{aligned}
$$

Ici, le terme $a_{2 j-3}(t)$ est négligeable devant $\left(a_{2 j-2}(t)+a_{2 j}(t)\right)^{-1} e^{w(t)}$ quand $t$ tend vers l'infini puisque ce dernier tend vers $+\infty$ (notez que $\left(a_{2 j-2}(t)+a_{2 j}(t)\right)^{-1} \geq 1 / 2$ ). Par conséquent,

$$
\left[a_{2 j-3}(t), a_{2 j-2}(t) e^{-w(t)}, a_{2 j-1}(t), a_{2 j}(t) e^{-w(t)}\right] \sim\left(a_{2 j-2}(t)+a_{2 j}(t)\right)^{-1} e^{w(t)} .
$$

De la même manière, on obtient

$$
\begin{aligned}
{\left[a_{2 j-4}(t) e^{-w(t)}, a_{2 j-3}(t), a_{2 j-2}(t)\right.} & \left.e^{-w(t)}, a_{2 j-1}(t)\right] \\
& \sim a_{2 j-4}(t) e^{-w(t)}+\left(a_{2 j-3}(t)+a_{2 j-1}(t)\right)^{-1} \\
& \sim\left(a_{2 j-3}(t)+a_{2 j-1}(t)\right)^{-1} .
\end{aligned}
$$

Finalement, par une récurrence évidente et en tenant compte de la parité des indices, il vient

et

$$
\begin{aligned}
x_{2 j}(t) & \sim\left(a_{2}(t)+\cdots+a_{2 j-2}(t)+a_{2 j}(t)\right)^{-1} e^{w(t)} \\
x_{2 j-1}(t) & \sim a_{1}(t)+a_{3}(t)+\cdots+a_{2 j-3}(t)+a_{2 j-1}(t) .
\end{aligned}
$$

Notez que les sommes ci-dessus sont, pour $j=N$, non nulles et convergent vers les nombres entiers que l'on a notés $a_{\text {pair }}$ et $a_{\text {impair }}$ plus haut. La démonstration du lemme est achevée.

Du lemme précédent et de la formule exprimant $\cosh (h)$, il vient

$$
h(t) \sim 2 \sqrt{a_{\text {pair }} a_{\text {impair }}} e^{-e^{t} w / 2} .
$$

De plus, on a facilement

$$
h^{*}(t) \sim 2 \sqrt{a_{\text {pair }} a_{\text {impair }}} e^{-e^{t} w / 2} .
$$

Algebraic 83 Geometric Topology, Volume 10 (2010) 
L'encadrement $h(t) \leq \ell(t) \leq h^{*}(t)$ permet de conclure

$$
\ell(t) \sim 2 \sqrt{a_{\text {pair }} a_{\text {impair }}} e^{-e^{t} w / 2} .
$$

La proposition 3.1 est démontrée.

\section{Divergence des lignes d'étirement élémentaires cylindriques}

Soient $t \mapsto g_{t}$ et $t \mapsto h_{t}$ deux lignes élémentaires de supports respectifs $\mu$ et $v$. Supposons que leurs feuilletages horocycliques $F_{\mu}(g)$ et $F_{\nu}(h)$ correspondent à la même multicourbe $\lambda=\lambda_{1} \cup \cdots \cup \lambda_{M}$ avec des pondérations respectives $w_{j}(g), w_{j}(h)$, $j \in\{1, \ldots, M\}$.

Posons $\delta_{j}(g, h)=\left(w_{j}(h)-w_{j}(g)\right) / 2$. D'après ce qui précède, on peut écrire

$$
\frac{\ell_{h_{t}}\left(\lambda_{j}\right)}{\ell_{g_{t}}\left(\lambda_{j}\right)} \sim K e^{-e^{t} \delta_{j}(g, h)},
$$

où $K$ est une constante strictement positive qui ne dépend que des laminations $\mu$ et $\nu$. Par conséquent, comme le rapport $\ell_{h_{t}}\left(\lambda_{j}\right) / \ell_{g_{t}}\left(\lambda_{j}\right)$ minore l'exponentielle de la distance $d_{\mathcal{T}}\left(g_{t}, h_{t}\right)$, on a

$$
\delta_{j}(g, h)<0 \Longrightarrow \lim _{t \rightarrow \infty} d_{\mathcal{T}}\left(g_{t}, h_{t}\right)=\infty
$$

En particulier, on obtient le résultat suivant:

Proposition 4.1 La distance de Thurston n'est pas symétrique le long d'une ligne d'étirement élémentaire cylindrique. Plus précisément, si $t \mapsto h_{t}$ est une ligne d'étirement élémentaire cylindrique, on a, pour tout $c>0$,

$$
\forall t, d_{\mathcal{T}}\left(h_{t}, h_{t+c}\right)=c \quad \text { et } \quad \lim _{t \rightarrow \infty} d_{\mathcal{T}}\left(h_{t+c}, h_{t}\right)=\infty .
$$

Démonstration L'égalité $d_{\mathcal{T}}\left(h_{t}, h_{t+c}\right)=c$ vient du fait que la ligne d'étirement est une géodésique. Pour établir la limite, notez que, pour tout $j \in\{1, \ldots, M\}$,

$$
\delta_{j}\left(h_{c}, h_{0}\right)=\left(w_{j}(h)-w_{j}\left(h_{c}\right)\right) / 2=w_{j}(h)\left(1-e^{c}\right) / 2<0 .
$$

Donc $\lim _{t \rightarrow \infty} d_{\mathcal{T}}\left(h_{t+c}, h_{t}\right)=\infty$.

Nous pouvons maintenant démontrer le résultat qui suit.

Proposition 4.2 Deux lignes d'étirement élémentaires dont les directions sont des classes projectives distinctes de la même multicourbe divergent. 
Démonstration Considérons deux lignes d'étirement comme dans l'énoncé de la proposition. Notons $\mu$ et $v$ leurs supports. Fixons un point base $g$ sur la ligne d'étirement de support $\mu$ et un point base $h$ sur la ligne d'étirement de support $v$. Par hypothèse, les directions des deux lignes sont des classes projectives associées à la même multicourbe $\lambda=\lambda_{1} \cup \cdots \cup \lambda_{M}$. Notons $\left(w_{1}, \ldots, w_{M}\right)$ et $\left(w_{1}^{\prime}, \ldots, w_{M}^{\prime}\right)$ les pondérations sur les composantes de $\lambda$ qui coïncident avec les largeurs des cylindres des feuilletages horocycliques $F_{g}(\mu)$ et $F_{h}(v)$ respectivement. Par hypothèse, les vecteurs $\left(w_{1}, \ldots, w_{M}\right)$ et $\left(w_{1}^{\prime}, \ldots, w_{M}^{\prime}\right)$ ne sont pas colinéaires.

Fixons la paramétrisation $t \mapsto g_{t}$ pour la ligne d'étirement passant par $g$ et de support $\mu$ de telle sorte que $g_{0}=g$. Pour montrer que les lignes divergent, il suffit de trouver une paramétrisation $t \mapsto h_{t}$ de la ligne passant par $h$ et de support $v$ telle que les distances $d_{\mathcal{T}}\left(g_{t}, h_{t}\right)$ et $d_{\mathcal{T}}\left(h_{t}, g_{t}\right)$ tendent vers l'infini lorsque $t$ tend vers $+\infty$. Cela revient à choisir le point base $h_{0}$ de la paramétrisation.

D'après ce qui précède, il suffit de montrer qu'il existe un point base $h_{0}$ et deux indices $j_{0}, j_{1} \in\{1, \ldots, M\}$ tels que $\delta_{j_{0}}\left(g, h_{0}\right)=\left(w_{j_{0}}^{\prime}\left(h_{0}\right)-w_{j_{0}}\right) / 2<0$ et $\delta_{j_{1}}\left(h_{0}, g\right)=$ $\left(w_{j_{1}}-w_{j_{1}}^{\prime}\left(h_{0}\right)\right) / 2<0$. Pour tout $j \in\{1, \ldots, M\}$, il existe un unique $u \in \mathbb{R}$ tel que $w_{j}^{\prime}\left(h_{0}\right)=e^{u} w_{j}^{\prime}$. Ainsi, nous sommes ramenés à prouver l'existence d'un nombre réel $u$ et de deux indices $j_{0}, j_{1} \in\{1, \ldots, M\}$ tels que $e^{u} w_{j_{0}}^{\prime}<w_{j_{0}}$ et $e^{u} w_{j_{1}}^{\prime}>w_{j_{1}}$.

On peut sans perte de généralité supposer que $M=2$ et que, quitte à modifier les points base $g$ et $h$, on ait $w_{1}=w_{1}^{\prime}=1$. Posons $w_{2}=w$ et $w_{2}^{\prime}=w^{\prime}$. La non colinéarité des vecteurs $(1, w)$ et $\left(1, w^{\prime}\right)$ signifie que le rapport $w^{\prime} / w$ n'est pas égal à un. Quitte à échanger les rôles des lignes d'étirement, on peut supposer que $w^{\prime} / w>1$. On cherche donc un nombre réel $u$ tel que

$$
\log \left(e^{u} / 1\right) \log \left(e^{u} w^{\prime} / w\right)<0,
$$

c'est-à-dire tel que

$$
u\left(u+\log \left(w^{\prime} / w\right)\right)<0 .
$$

Il est facile de voir que n'importe quel nombre $u$ dans l'intervalle $]-\log \left(w^{\prime} / w\right), 0[$ convient. La démonstration est achevée.

\section{Divergence des lignes cylindriques}

Nous passons maintenant au cas général, où $\mu$ n'est plus nécessairement élémentaire.

Théorème 5.1 Soit $\mu$ une lamination géodésique complète récurrente par chaînes. Considérons une ligne d'étirement cylindrique, $t \mapsto h_{t}$, de support $\mu$ et de direction la classe projective d'une multicourbe $\lambda$ équipée d'une pondération fixée. Si $\lambda_{j}$ désigne 
une composante de $\lambda$ et $w_{j}$ le poids associé, on a l'estimation suivante, quand $t$ tend vers l'infini,

$$
\ell_{h_{t}}\left(\lambda_{j}\right) \sim 2 \sqrt{K_{j}} e^{-e^{t} w_{j} / 2}
$$

où $K_{j} \in \mathbb{N}$ est le nombre de régions non-feuilletées du feuilletage horocyclique adjacentes à un bord du cylindre de cœur $\lambda_{j}$ multiplié par le nombre de régions nonfeuilletées adjacentes à l'autre bord.

Avant de commencer la démonstration, faisons quelques remarques. Soit $C$ un cylindre du feuilletage horocyclique, de largeur $w(t)=e^{t} w$. Le cylindre $C$ est traversé en général par un nombre indénombrable de feuilles de $\mu$ et il n'y a pas de notion canonique de décomposition en bandes dans ce cas. Nous allons utiliser une approximation finie de $\mu$ et sa décomposition en bandes.

Avant cela, montrons qu'il existe une unique feuille du feuilletage horocyclique de $C$ dont la longueur est minimale. Tout d'abord, la longueur d'une feuille $\alpha$ du feuilletage horocyclique est la somme des longueurs des arcs d'horocycles de $\alpha \backslash \mu$, car la lamination $\mu$ est de mesure de Lebesgue nulle. Ensuite, le nombre de ces arcs d'horocycles est dénombrable et la longueur de $\alpha$ ne dépend pas de l'ordre dans lequel ces arcs apparaissent. Ainsi, quitte à réorganiser les arcs d'horocycles, on peut supposer que le cylindre est composé de deux bandes et que la feuille $\alpha$ est composée de deux arcs d'horocycles dont les convexités sont de signes opposés. Nous avons vu précédemment qu'il y a dans ce cas un unique minimum $h^{*}(t)$ valant

$$
h^{*}(t)=2 \sqrt{a_{\text {pair }}(t) a_{\text {impair }}(t)} e^{-w(t) / 2},
$$

où $a_{\text {pair }}(t)$ et $a_{\text {impair }}(t)$ désignent les épaisseurs des deux bandes.

On appelle tronçon une bande minimale d'épaisseur 1, c'est-à-dire une bande minimale dont le bord contient le côté d'une région non-feuilletée.

Considérons l'approximation suivante du cylindre $C$. Pour tout $t$, soit $C^{\prime}$ le cylindre obtenu en ne gardant du cylindre $C$ que les tronçons et en recollant les bords géodésiques de ces tronçons par des isométries. Notez que la largeur de $C^{\prime}$ est également $w(t)=e^{t} w$. Notons $h^{\prime}(t)$ la hauteur du cylindre $C^{\prime}$ et $h(t)$ la hauteur du cylindre $C$.

Démonstration du théorème 5.1 La démonstration repose sur l'inégalité suivante:

$$
\forall t, h^{\prime}(t) \leq h(t)
$$

Cette inégalité à été établie dans [5, page 398, Lemma 2.8]. Sa preuve consiste à remarquer que si l'on insère une bande à $C^{\prime}$ pour obtenir un cylindre $C^{\prime \prime}$, la nouvelle hauteur $h^{\prime \prime}(t)$ est strictement plus grande que celle de $C^{\prime}$. Un petit dessin dans le demi-plan supérieur comme celui de [5] permet de s'en convaincre. 
On a les encadrements

$$
h^{\prime}(t) \leq h(t) \leq \ell(t) \leq h^{*}(t),
$$

où $\ell(t)$ désigne la longueur du cœur du cylindre $C$ pour la métrique $h_{t}$.

On a vu précédemment que les termes $h^{\prime}(t)$ et $h^{*}(t)$ sont tous deux équivalents, lorsque $t$ tend vers l'infini, à

$$
2 \sqrt{K} e^{-w(t) / 2},
$$

où $K \in \mathbb{N}$ est le nombre de régions non-feuilletées adjacentes à un côté de $C$ multiplié par le nombre de régions non-feuilletées adjacentes à l'autre côté de $C$. La démonstration est terminée.

On en déduit immédiatement, par les mêmes démonstrations, les versions générales des théorèmes établis dans le cadre des lignes d'étirement élémentaires.

Théorème 5.2 Deux lignes d'étirement cylindriques dont les directions correspondent à des classes projectives distinctes de la même multicourbe divergent.

Théorème 5.3 Soit $t \mapsto h_{t}$ une ligne d'étirement cylindrique. Pour tout nombre $c>0$, on a

$$
\forall t, d_{\mathcal{T}}\left(h_{t}, h_{t+c}\right)=c \quad \text { et } \quad \lim _{t \rightarrow \infty} d_{\mathcal{T}}\left(h_{t+c}, h_{t}\right)=\infty
$$

Remarque 5.4 Considérons plusieurs composantes $\lambda_{j}, j \in J \subset\{1, \ldots, M\}$ de $\lambda$. Posons $w_{\min }:=\inf _{j \in J}\left\{w_{j}\right\}$ et $J_{\min }:=\left\{j \in J: w_{j}=w_{\min }\right\}$. On obtient l'estimation suivante, quand $t$ tend vers l'infini,

$$
\ell_{h_{t}}\left(\bigcup_{j \in J} \lambda_{j}\right) \sim 2\left(\sum_{j \in J_{\min }} \sqrt{K_{j}}\right) e^{-e^{t} w_{\min } / 2}
$$

\section{Le problème des lignes parallèles et divergentes}

Nous venons de démontrer que deux lignes cylindriques de directions différentes mais topologiquement égales divergent. En fait, on a le résultat général suivant.

Proposition 6.1 Deux lignes d'étirement cylindriques sont parallèles seulement si elles ont la même direction.

Démonstration Montrons la contraposée, c'est-à-dire que deux lignes d'étirement cylindriques dont les directions sont différentes ne sont pas parallèles. Notons $\lambda$ et $\lambda^{\prime}$ les directions de deux lignes d'étirement cylindriques avec $\lambda \neq \lambda^{\prime}$. Les objets $\lambda$ et $\lambda^{\prime}$ 
sont donc les classes projectives de multicourbes pondérées. Supposons d'abord que les directions $\lambda$ et $\lambda^{\prime}$ sont topologiquement distinctes. Le théorème principal de [4] affirme qu'on a, le long d'une ligne d'étirement $t \mapsto h_{t}$ et de direction $\lambda$, les comportements asymptotiques suivants. Soit $\alpha$ une lamination géodésique mesurée. La longueur $\ell_{h_{t}}(\alpha)$ de $\alpha$

- converge vers 0 si $\alpha \subset \lambda$,

- converge vers l'infini, si $i(\alpha, \lambda) \neq 0$,

- est bornée dans $\mathbb{R}_{+}^{*}$, si $\alpha \cap \lambda=\varnothing$.

Par conséquent, si $t \mapsto g_{t}$ et $t \mapsto h_{t}$ sont les lignes d'étirement de directions $\lambda$ et $\lambda^{\prime}$, les rapports

$$
\frac{\ell_{h_{t}}(\lambda)}{\ell_{g_{t}}(\lambda)} \text { et } \frac{\ell_{g_{t}}\left(\lambda^{\prime}\right)}{\ell_{h_{t}}\left(\lambda^{\prime}\right)}
$$

convergent vers l'infini, ce qui montrent que les lignes divergent. Cette conclusion ne dépend pas de la paramétrisation positive par la longueur d'arc choisie. Les lignes ne sont donc pas parallèles.

Supposons donc que les directions $\lambda$ et $\lambda^{\prime}$ soient topologiquement identiques. Le théorème 5.2 permet de conclure que les lignes divergent alors. La démonstration est achevée.

Nous allons maintenant établir la réciproque.

Théorème 6.2 Deux lignes d'étirement cylindriques sont parallèles si et seulement si elles ont la même direction.

Démonstration Il reste à montrer que deux lignes d'étirement cylindriques de mêmes directions sont parallèles. Considérons une géodésique simple fermée $\alpha$ et une ligne d'étirement $t \mapsto h_{t}$ de direction $\lambda$. Notons $C_{1}, \ldots, C_{M}$ les cylindres associés aux composantes $\lambda_{1}, \ldots, \lambda_{M}$ de $\lambda$. Fixons également dans chaque cylindre un arc géodésique $\rho_{j}$ de longueur $w_{j}(t), j=1, \ldots, M$. La courbe $\alpha$ traverse les cylindres et effectue un certain nombre de tours autour des composantes de $\lambda$. Nous avons donc l'encadrement suivant

$$
\sum_{j=1}^{M}\left|\alpha \cap \lambda_{j}\right| w_{j}(t) \leq \ell_{h_{t}}(\alpha) \leq \sum_{j=1}^{M}\left(\left|\alpha \cap \lambda_{j}\right| w_{j}(t)+\left|\alpha \cap \rho_{j}\right| \ell_{h_{t}}\left(\lambda_{j}\right)\right) .
$$

Cet encadrement montre que si $i(\alpha, \lambda) \neq 0$, la longueur $\ell_{h_{t}}(\alpha)$ est de l'ordre de $e^{t}$. 
On en déduit que, si $t \mapsto g_{t}$ est une ligne d'étirement de direction $\lambda$, les rapports $\ell_{h_{t}}(\alpha) / \ell_{g_{t}}(\alpha)$ et $\ell_{g_{t}}(\alpha) / \ell_{h_{t}}(\alpha)$, avec $i(\alpha, \lambda) \neq 0$, sont bornés. Dans le cas où $\alpha \cap \lambda=\varnothing$, le théorème de l'article [4] déjà cité dans la démonstration précédente implique également que les rapports $\ell_{h_{t}}(\alpha) / \ell_{g_{t}}(\alpha)$ et $\ell_{g_{t}}(\alpha) / \ell_{h_{t}}(\alpha)$ sont bornés. Finalement, on a montré plus haut dans le théorème 5.1 que si $\alpha \subset \lambda$, les rapports sont eux aussi bornés.

Nous venons donc d'établir que pour toute multicourbe $\alpha$, il existe un nombre $M(\alpha)>0$ tel que, pour tout $t \geq 0$,

$$
\frac{1}{M(\alpha)} \leq \frac{\ell_{h_{t}}(\alpha)}{\ell_{g_{t}}(\alpha)} \leq M(\alpha)
$$

On conclut la démonstration avec la proposition qui suit, en se souvenant qu'une ligne d'étirement converge vers sa direction [3].

Proposition 6.3 Soient $\left(g_{n}\right)$ et $\left(h_{n}\right)$ deux suites de l'espace de Teichmüller convergeant vers le même point de la compactification $\mathcal{T}(\Sigma) \cup \mathcal{P} \mathcal{L}(\Sigma)$ de l'espace de Teichmüller et telles que, pour toute courbe simple fermée $\alpha$, il existe un nombre $M(\alpha)>0$ tel que, pour tout $n \in \mathbb{N}$,

$$
\frac{1}{M(\alpha)} \leq \frac{\ell_{h_{n}}(\alpha)}{\ell_{g_{n}}(\alpha)} \leq M(\alpha) .
$$

Alors les suites $\left(d_{\mathcal{T}}\left(g_{n}, h_{n}\right)\right)$ et $\left(d_{\mathcal{T}}\left(h_{n}, g_{n}\right)\right)$ sont bornées.

Démonstration On raisonne par l'absurde. Supposons que l'une des suites, disons $\left(d_{\mathcal{T}}\left(g_{n}, h_{n}\right)\right)$, ne soit pas bornée. Quitte à extraire une sous-suite, on peut supposer que $\left(d_{\mathcal{T}}\left(g_{n}, h_{n}\right)\right)$ converge vers l'infini. Cela implique que pour tout $n$, il existe une géodésique simple fermée $\alpha_{n}$ telle que

$$
\frac{\ell_{h_{n}}\left(\alpha_{n}\right)}{\ell_{g_{n}}\left(\alpha_{n}\right)} \geq n
$$

Quitte à extraire une sous-suite, on peut supposer que la suite des classes projectives $\left(\left[\alpha_{n}\right]\right)$ converge dans $\mathcal{P} \mathcal{L}(\Sigma)$ vers la classe projective $\left[\alpha_{\infty}\right]$. De plus, comme les suites $\left(g_{n}\right)$ et $\left(h_{n}\right)$ convergent, par hypothèse, vers la même limite dans $\mathcal{P} \mathcal{L}(\Sigma)$, il existe deux suites $\left(x_{n}\right)$ et $\left(y_{n}\right)$ de réels positifs telles que

$$
\forall \beta \in \mathcal{M L}(\Sigma), \lim _{n \rightarrow \infty} \frac{y_{n} \ell_{h_{n}}(\beta)}{x_{n} \ell_{g_{n}}(\beta)}=1 .
$$

Par hypothèse, on a, pour $\alpha$ donné,

$$
\frac{y_{n}}{x_{n}} \frac{1}{M(\alpha)} \leq \frac{y_{n} \ell_{h_{n}}(\alpha)}{x_{n} \ell_{g_{n}}(\alpha)} \leq \frac{y_{n}}{x_{n}} M(\alpha)
$$

Algebraic $\mathcal{G}$ Geometric Topology, Volume 10 (2010) 
On en déduit que la suite $\left(y_{n} / x_{n}\right)$ est bornée dans $\mathbb{R}_{+}^{*}$. Quitte à extraire une sous suite, on peut supposer que cette suite converge vers un nombre $c \in \mathbb{R}_{+}^{*}$.

Par continuité de la fonctionnelle d'intersection sur l'espace des courants géodésiques [1], on a

$$
\lim _{n \rightarrow \infty} \frac{y_{n} \ell_{h_{n}}\left(\alpha_{n}\right)}{x_{n} \ell_{g_{n}}\left(\alpha_{n}\right)}=1 \text {, soit } \lim _{n \rightarrow \infty} \frac{\ell_{h_{n}}\left(\alpha_{n}\right)}{\ell_{g_{n}}\left(\alpha_{n}\right)}=\frac{1}{c} .
$$

Nous obtenons une contradiction, ce qui montre que les suites $\left(d_{\mathcal{T}}\left(g_{n}, h_{n}\right)\right)$ et $\left(d_{\mathcal{T}}\left(h_{n}, g_{n}\right)\right)$ sont bornées.

\section{Références}

[1] F Bonahon, The geometry of Teichmüller space via geodesic currents, Invent. Math. 92 (1988) 139-162 MR931208

[2] H Masur, On a class of geodesics in Teichmüller space, Ann. of Math. (2) 102 (1975) 205-221 MR0385173

[3] A Papadopoulos, On Thurston's boundary of Teichmüller space and the extension of earthquakes, Topology Appl. 41 (1991) 147-177 MR1135095

[4] G Théret, On Thurston's stretch lines in Teichmüller space, Preprint (2005)

[5] G Théret, On the negative convergence of Thurston's stretch lines towards the boundary of Teichmüller space, Ann. Acad. Sci. Fenn. Math. 32 (2007) 381-408 MR2337484

[6] WP Thurston, Minimal stretch maps between hyperbolic surfaces arXiv: math/9801039

Max Planck Institut für Mathematik

Vivatsgasse 7, 53100 Bonn, Germany

theret@mpim-bonn.mpg.de

Received: 24 April $2010 \quad$ Revised: 30 June 2010 